12

\title{
Особенности применения теоремы вириала для магнитных систем с квазибессиловыми обмотками
}

\author{
(C) Г.А. Шнеерсон, ${ }^{1}$ С.Л. Шишигин ${ }^{2}$ \\ ${ }^{1}$ Санкт-Петербургский политехнический университет Петра Великого, \\ 195251 Санкт-Петербург, Россия \\ ${ }^{2}$ Вологодский государственный университет, \\ 160000 Вологда, Россия \\ e-mail: gashneerson@mail.ru, ctod28@yanex.ru
}

Поступило в Редакцию 6 сентября 2021 г.

В окончательной редакции 10 октября 2021 г.

Принято к публикации 11 октября 2021 г.

Показано, что в магнитной системе с тонкостенной уравновешенной обмоткой, близкой к бессиловой, возможно существенное увеличение параметра $\theta=W_{M} \gamma / M \sigma_{M}$, который, согласно теореме вириала, характеризует отношение энергии магнитной системы $W_{M}$ к массе оборудования с плотностью материала $\gamma$, в котором под действием электромагнитных сил имеет место механическое напряжение $\sigma_{M}$. В квазибессиловой магнитной системе основная часть обмотки находится в состоянии локального равновесия и лишь в относительно небольшой части оборудования имеет место напряженное состояние. Эта часть определяет массу всей системы, и эта масса может быть минимизирована. Построены конфигурации уравновешенных тонкостенных обмоток, на границах которых выполнены одновременно два граничных условия - отсутствие нормальной к границе компоненты индукции и постоянство произведения индукции на радиус. Рассмотрен пример системы, состоящей из основной части - последовательности уравновешенных „поперечных“ модулей в форме плоских дисков, и концевых частей, состоящих из комбинации „поперечных“ модулей и „продольных“, имеющих вид вытянутых вдоль оси колец с уравновешенными торцевыми частями. Показано, что в рассмотренной системе характерный безразмерный параметр $\theta$ при неограниченном росте числа элементов основной части может достигать значения около 24 , а при изменении числа этих элементов в пределах 20-40 изменяется от 6 до 9.

Ключевые слова: квазибессиловое магнитное поле, энергия поля, масса магнитной системы, теорема вириала, минимизация отношения массы к энергии.

DOI: $10.21883 / J T F .2022 .02 .52022 .249-21$

\section{Введение}

В магнитной системе, находящейся в состоянии равновесия, равна нулю объемная сила, равная сумме электромагнитной силы и дивергенции тензора упругих напряжений. Вычисления, приведенные в работах [1-3], приводят к следующему выражению, связывающему энергию поля магнитной системы $W_{M}$ с интегралом от тензора упругих напряжений $S_{i i}$ (теорема вириала):

$$
W_{M}=\int \frac{B^{2}}{2 \mu_{0}} d V=-\int_{V} S_{i i} d V .
$$

Здесь первый из интегралов берется по всей неограниченной области. Второй охватывает область $V$, в которой отличны от нуля компоненты тензора упругих напряжений. Сечение, а значит, объем и масса магнитной системы определяются уровнем допустимых механических напряжений в элементах магнитной системы, нагруженных электромагнитными силами.

Уравнение (1) использовано в книге Лонгмайра [2] и в других работах для оценки отношения энергии к массе магнитной системы. В простейшем случае эти оценки исходят из допущения, что нагрузка, создаваемая электромагнитными силами, воспринимается лишь проводниками с током, создающими поле. Если при этом материал всех проводников имеет одинаковую плотность $\gamma$, из приведенного равенства (1) следует отношение энергии магнитного поля к массе обмотки $M=\gamma V$ :

$$
\frac{W}{M}=\theta \frac{\sigma}{\gamma},
$$

где $\sigma$ - величина, характеризующая напряженное состояние материала обмотки, $\theta-$ характерный безразмерный параметр (вириальный коэффициент).

В конкретных системах отношение энергии к массе рассчитывается с учетом их конструктивных особенностей. Например, расчеты безмоментной тонкостенной обмотки, в которых в качестве $\sigma$ использовалось значение касательной компоненты напряжения, дают значение $\theta=0.61$ [4]. Широко распространено мнение, что вириальный коэффициент $\theta$ не превышает единицу. Это утверждение в общем виде содержится в книге [2]. Оно подтверждено расчетами конкретных систем в работах [5-7] и данными, приведенными в обширном обзоpe [8]. В работах японских исследователей [9-12] показа- 
но, что при размещении проводников под определенным углом на тороидальной поверхности можно достигнуть интегрального равновесия в осевом или радиальном направлении. Локальное равновесие в рассмотренных системах отсутствует, нагрузка воспринимается проводниками, и вириальный параметр $\theta$ близок к единице.

В общем случае масса магнитной системы определяется как проводниками с током, так и другими конструктивными элементами, обеспечивающими ее прочность, например, бандажами. В последние годы в ряде работ исследованы возможности построения локально уравновешенных (квазибессиловых) тонкостенных обмоток. Обзор этих работ содержится в книге [13]. Применение таких обмоток дает возможности построения магнитных систем с уменьшенной массой нагруженных элементов. Идеальной является строго бессиловая система. Однако в работах $[2,3]$ показано, что магнитная система конечных размеров с бессиловым полем должна иметь границы, подверженные воздействию электромагнитных сил. Поэтому задача повышения отношения энергии к массе в системах с квазибессиловыми обмотками включает в себя две части. Первая - это создание магнитной системы максимально приближенной к бессиловой. Вторая - минимизация массы тех элементов, которые остаются нагруженными электромагнитными силами. Цель настоящей работы - показать, как могут быть построены магнитные системы с квазибессиловыми обмотками, позволяющие увеличить отношение энергии магнитного поля к массе и достичь значений вириального коэффициента $\theta$, много большего единицы.

\section{1. Исходные положения. Проблема минимизации массы неуравновешенной части магнитной системы}

Близкими к строго бессиловым являются тонкостенные квазибессиловые обмотки, находящиеся в состоянии локального равновесия, когда абсолютные значения индукции магнитного поля принимают одинаковые значения $B=|\mathbf{B}|$ по обе стороны обмотки. Остаточное эквивалентное напряжение в такой обмотке, рассчитанное по формуле Мизеса, характеризуется значением $\sigma_{M}=\eta B^{2} /\left(2 \mu_{0}\right)$. В локально уравновешенной тонкостенной обмотке прочностной коэффициент $\eta$ может принимать значение, много меньшее единицы. При этом число $\eta$ не зависит от толщины обмотки $\Delta$, а определяется отношением $\Delta / R$, где $R$ - радиус кривизны поверхности, на которой размещена обмотка. В частности, в однослойной цилиндрической обмотке при условии $\Delta / R \approx 0.1$ имеем $\eta \approx 0.2$ [14]. Сечение и, следовательно, масса уравновешенных проводников в локально уравновешенной обмотке определяется не прочностью материала, а другими факторами, например, критической плотностью тока. В качестве предельного случая в настоящей работе будет рассмотрена система с

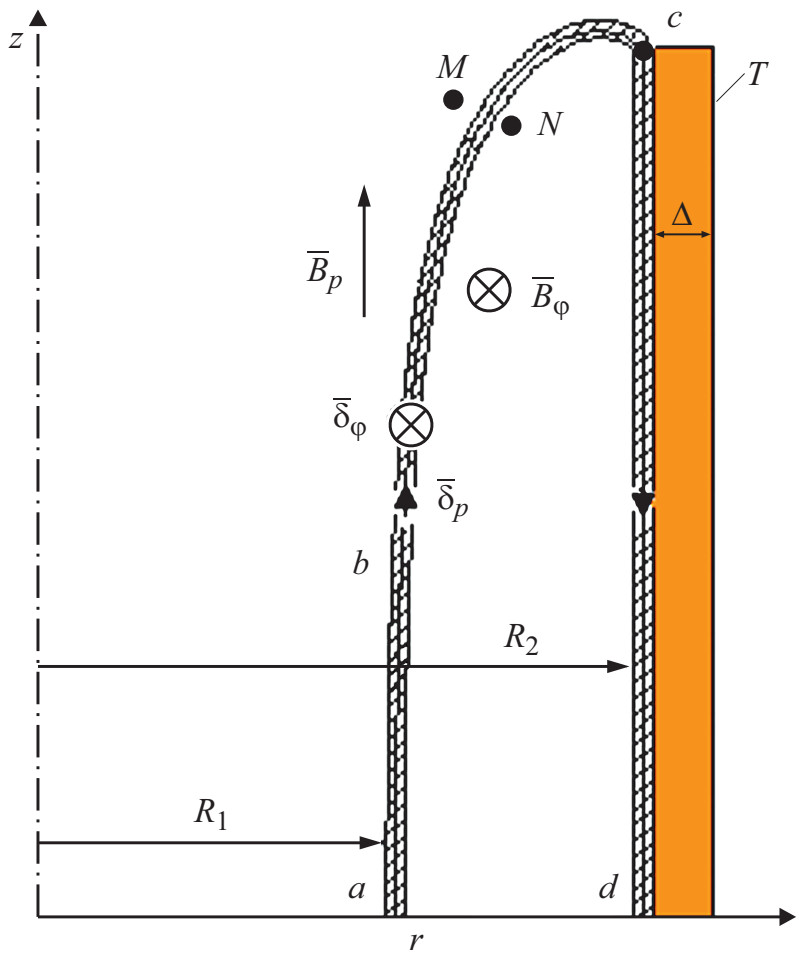

Рис. 1. Полубесконечная частично уравновешенная тонкостенная обмотка с внешним бандажом $T$.

квазибессиловой обмоткой, у которой толщина проводников мала настолько, что массой такой обмотки можно пренебречь по сравнению с массой элементов системы, подверженных воздействию электромагнитных сил.

Примером аксиально симметричной магнитной системы с частично уравновешенной границей является показанная на рис. 1 конфигурация в виде вытянутого вдоль оси („продольного“) модуля, имеющего форму цилиндра с внутренним радиусом $R_{1}$ и внешним $R_{2}$ с размещенной на нем тонкой обмоткой [13]. На всей ее границе должна быть равна нулю азимутальная компонента электромагнитной силы, следовательно, должна отсутствовать нормальная компонента индукции полоидального поля. Это имеет место, если выполнено условие

$$
\psi_{P}=r A_{\phi}=\mathrm{const}=C_{1},
$$

где $\psi_{P}-$ функция потока полоидального поля, $A_{\varphi}$ - азимутальная компонента векторного потенциала. На уравновешенной части тонкостенной границы $a b c$ (рис. 1) абсолютные значения индукции полоидального поля в точке $M$ и индукции азимутального поля в точке $N$ должны быть равны

$$
B_{P}(M)=B_{P}(N)=\mu_{0}\left|i_{P}\right| /(2 \pi r)=\mu_{0}\left|\psi_{i}\right| / r,
$$

где $i_{P}$ - полоидальный ток в обмотке, $\psi_{i}=\mu_{0} i_{P} / 2 \pi$ функция полоидального тока, $r-$ радиальная координата точки $N$. Таким образом, на этом участке должно быть выполнено второе граничное условие:

$$
r B_{P}=\mu_{0}\left|\psi_{i}\right|=\text { const }=C_{2} .
$$


Построение поля с упомянутыми двумя граничными условиями является примером нелинейной задачи, поскольку форма границы не задана, а формируется в процессе решения. Аналогичные задачи рассматриваются в электростатике, в теории струй идеальной жидкости. Их отличие состоит в том, что в рассматриваемой задаче на границах расчетной области постоянными являются значения произведения $r B_{p}$, тогда как в задачах электростатики на части границы задается постоянство напряженности электрического поля, а в теории струй - постоянство скорости течения. Описанию течений со свободными границами в гидромеханике, построению электродов с постоянной напряженностью поля в электростатике посвящено множество работ и ряд монографий, например [15-19]. При решении таких задач с плоским полем эффективно используется метод конформных преобразований. Результаты этих расчетов могут быть использованы в расчетах осесимметричных систем с бессиловыми обмотками на удаленных от оси участках границы, где поле близко к плоскому.

В настоящей работе для построения конфигураций обмоток, удовлетворяющих поставленным условиям, использован метод, описанный в работе [19]. Краткое описание процедуры построения фигур со свободными границами методом итераций приведено в Приложении.

Показанный на рис. 1 пример демонстрирует упомянутую выше характерную особенность магнитных систем с бессиловым полем: такая система не может быть полностью бессиловой [2,3]. В частности, аналитические решения плоских задач показывают, что в общем случае оба граничные условия могут иметь место лишь на части границы. Например, напряженность электрического поля может быть постоянна на участке в окрестности кромки электрода $[16,17]$. Лишь в отдельных случаях удается построить фигуру, на всей границе которой выполняются оба условия. В аксиально симметричных двумерных конфигурациях дополнительным фактором является различный закон убывания напряженности полоидального и азимутального полей при увеличении радиуса: $1 / r^{2}$ в первом и $1 / r-$ во втором случаях.

Формирование границы методом итераций показало, что фигуру с равновесным участком, показанную на рис. 1, удается построить, если отношение внешнего радиуса $R_{2}$ к внутреннему $R_{1}$ не превышает значения $A=R_{2} / R_{1} \sim 1.64$. У магнитной системы большой длины имеет место следующее выражение для суммарной энергии полоидального и азимутального полей в расчете на единицу длины: $W_{M}^{\prime} \sim \pi R_{1}^{2}(1 / 2+\ln A) B_{0}^{2} / \mu_{0}$, где $B_{0}-$ индукция однородного полоидального поля на оси магнита большой длины. К уравновешенной границе $a b c$, включающей в себя часть внутренней цилиндрической границы и ее торцевой участок, примыкает неуравновешенная внешняя цилиндрическая часть обмотки $c d$, на которой выполнено условие постоянства функций $\psi_{P}$ и $\psi_{i}$, но не выполнено условие (4). Пренебрегая магнитным давлением полоидалного поля, можно принять, что на неуравновешенную часть обмотки воздействует магнитное давление $P_{M}=\left(1 / 2 \mu_{0}\right) B_{\varphi}^{2}$ где $B_{\phi}=B_{0} / A-$ индукция азимутального поля. Для удержания этой части обмотки требуется цилиндрический бандаж $T$ (рис. 1). Под действием магнитного давления в тонком бандаже толщиной $\Delta \ll R_{2}$, удерживающем тонкую обмотку, имеет место азимутальное механическое напряжение $\sigma_{M} \approx \sigma_{\phi}=P_{M} R_{2} / \Delta$. Отсюда находим толщину и массу бандажа (в расчете на единицу длины) $\Delta_{r}=P_{M} R_{2} / \sigma_{M}$, $M^{\prime}=2 \pi R_{2} \Delta \gamma=2 \pi R_{2}^{2} P_{M} \gamma / \sigma_{M}$. Далее находим значение отношения энергии к массе

$$
\frac{W_{M}^{\prime}}{M^{\prime}} \approx \frac{\sigma_{M}}{\gamma}\left(\frac{1}{2}+\ln A\right) .
$$

В соответствии с формулой (2) вириальный коэффициент принимает значение $\theta=\gamma W_{M}^{\prime} / \sigma_{M} M^{\prime} \sim 1 / 2+\ln A$. Предельное значение этого параметра для системы, представленной на рис. 1 , есть $\theta \sim 0.5+\ln 1.64 \sim 1$.

В книге [14] приведены результаты расчетов конфигурации полубесконечного модуля, расположенного в зазоре между двумя коаксиальными цилиндрами с радиусами $R_{0}$ и $R_{1}$ с заданным на них магнитным потенциалом, равным нулю (рис. 2, $a$ ). Расчеты методом итераций (Приложение) показали, что при заданном значении отношения $R_{1} / R_{0}$ существует лишь одна фигура с внутренним радиусом $R_{i}$ и внешним $R_{e}$, на всей границе которой поддерживаются постоянными произведения $r A_{\varphi}$ и $r B_{p}$.

На рис. 2, $b$ приведены зависимости отношения $\left(R_{1}-R_{0}\right) /\left(R_{i}-R_{0}\right)$ и аспектного отношения $A=R_{e} / R_{i}$ от параметра $R_{0} / R_{1}$. В предельном случае $R_{0} / R_{1}=0$, когда уравновешенная обмотка находится в коаксиальном цилиндрическом экране, упомянутые граничные условия могут быть выполнены на всей границе лишь при определенном соотношении радиусов: $R_{i} / R_{e} / R_{2} \sim 1 / 2.12 / 2.57$ [14]. При этом $R_{1}^{2}=R_{e}^{2}+R_{i} R_{e}$. $\mathrm{B}$ расчете на единицу длины энергия магнитного поля этой системы составляет $W_{M}^{\prime}=\pi R_{0}^{2} \times$ $\times[1+1 / A+2 \ln A] B_{0}^{2} / 2 \mu_{0}$. В этой системе неуравновешенным элементом является короткозамкнутый диамагнитный экран. ${ }^{1}$ Он подвержен воздействию магнитного давления полоидального поля с индукцией $B\left(R_{1}\right)=R_{0} R_{i} / R_{e}$. Масса тонкостенного внешнего бандажа, воспринимающего магнитное давление на экран, составляет $M^{\prime}=\pi R_{i}^{2}(1+1 / A)\left(B_{0}^{2} / \mu_{0}\right) /\left(\gamma / \sigma_{M}\right)$. Параметр $\theta$ определяется по формуле

$$
\theta=\frac{1+1 / A+2 \ln A}{2(1+1 / A)} \sim 1
$$

где $A \sim 2.12$. Как и в предыдущем примере, $\theta \sim 1$. В предельном случае, когда выполнено условие $R_{1}-R_{0} \ll R_{0}$,

\footnotetext{
${ }^{1}$ Короткозамкнутый диамагнитный экран - это проводящий цилиндр, на поверхности которого выполнено условие равенства нулю магнитного потока. При практической реализации ток в экране, распределенный в соответствии с принятой моделью, может быть создан внешними источниками.
} 

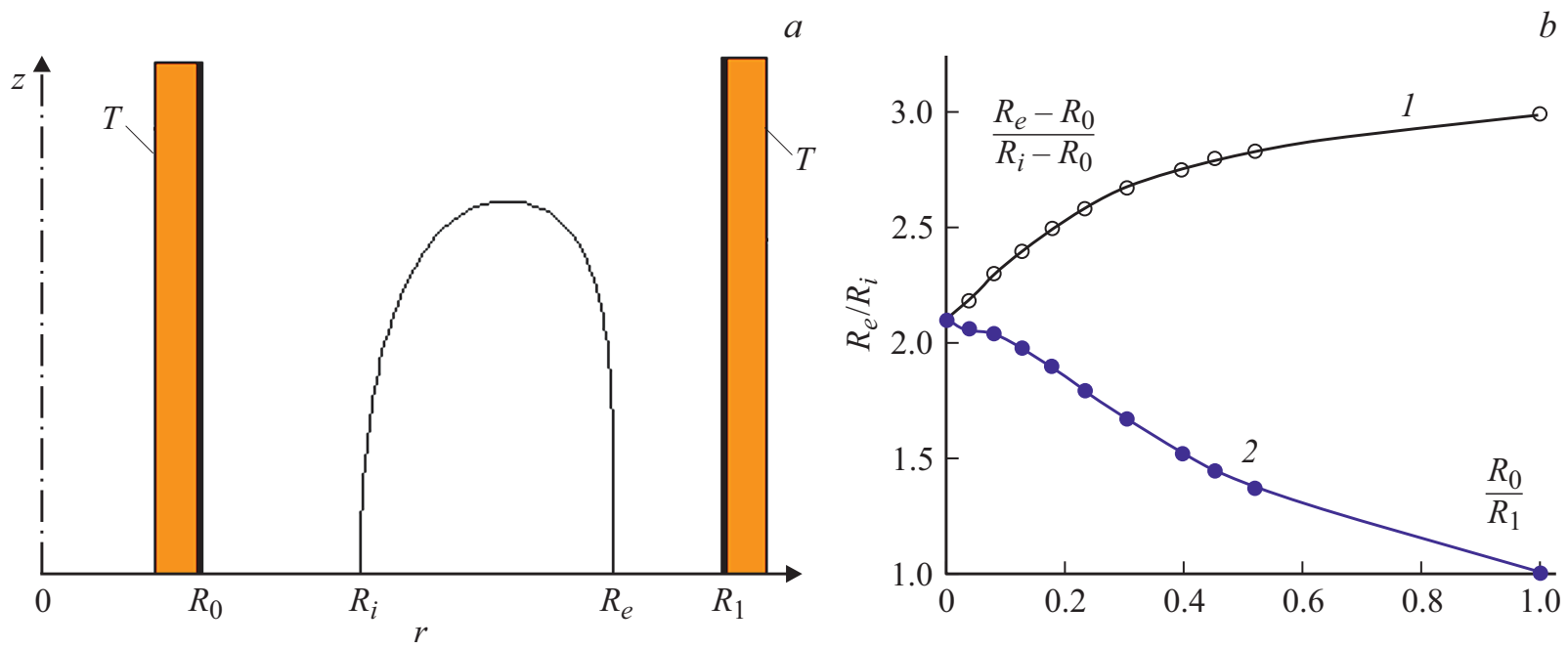

Рис. 2. $a-$ полностью уравновешенная тонкостенная обмотка большой длины (продольный модуль), расположенная в зазоре между двумя коаксиальными экранами; $b$ - зависимости, характеризующие параметры продольного уравновешенного модуля, расположенного между двух коаксиальных экранов: $1-\left(R_{e}-R_{0}\right) /\left(R_{i}-R_{0}\right)=f\left(R_{0} / R_{1}\right), 2-R_{e} / R_{i}=f\left(R_{0} / R_{1}\right)$.

и поле в зазоре близко к плоскому, для равновесных конфигураций рис. 2 выполняются соотношения: $R_{i}-R_{0}=R_{1}-R_{e}=\left(R_{1}-R_{0}\right) / 4,\left(R_{1}-R_{0}\right)=2\left(R_{e}-R_{1}\right)$.

$\mathrm{B}$ рамках модели тонкостенной бессиловой обмотки можно рассчитать конфигурацию линий тока на границах модулей. В работе [20] показано, что при известном значении отношения индукций азимутального и полоидального полей по обе стороны обмотки линии тока описываются уравнением, связывающим приращение азимутальной координаты точки на линии тока при изменении ее радиальной координаты от значения $r_{1}$ до значения $r_{2}$ и смещении на отрезок $d l_{p}$ в плоскости $r-z$ :

$$
\Delta \varphi=\int_{r_{1}}^{r_{2}} \frac{B_{p} d l_{p}}{B_{\varphi} r} .
$$

На уравновешенных участках угол между вектором линейной плотности тока и касательной к контуру продольного сечения тонкой обмотки в каждой точке составляет $45^{\circ}$. В этом случае вектор линейной плотности тока параллелен сумме равных по модулю векторов индукции полоидального и азимутального полей. Формула (8) в этом случае принимает вид

$$
\Delta \varphi=\int_{r_{1}}^{r_{2}} \frac{d l_{p}}{r} .
$$

На прямолинейных участках равновесных границ продольного модуля с радиусом $R$ линии тока имеют форму спирали: $\Delta z=R \Delta \varphi$. На плоской части границы $d l_{p}=d r$ и линии тока описываются уравнением логарифмической спирали

$$
\Delta \varphi=\int_{r_{1}}^{r_{2}} \frac{d r}{r}=\ln \frac{r_{2}}{r_{1}} .
$$

Во всех рассмотренных примерах с продольными модулями имеет место неуравновешенный элемент системы в виде цилиндра (бандажа или экрана). Длина этого цилиндра мало отличается от длины уравновешенного модуля. Поэтому вириальный параметр $\theta$ в этих системах близок к единице. Радикальным способом увеличения этого параметра является резкое снижение массы элементов, воспринимающих неуравновешенную радиальную силу. Для этого должна быть сокращена длина этих элементов и снижены воздействующие на них силы.

\section{2. Отношение энергии к массе в системе уравновешенных поперечных модулей}

Резкое снижение массы неуравновешенных элементов возможно в магнитной системе, состоящей из набора чередующихся поперечных модулей. Два варианта такой системы представлены на рис. 3 и 4. Каждый поперечный модуль - это тонкостенная локально уравновешенная обмотка, имеющая форму тела вращения, аксиальный размер которого много меньше внешнего радиуса. В системе неограниченной длины они образуют периодическую структуру с шагом $h$. Реальная система конечной длины помимо набора чередующихся поперечных модулей включает в себя две концевые части магнитной системы. В разд. 3 показано, что эти части могут быть построены так, что граничные условия в системе с конечным числом поперечных модулей остаются такими же, как в системе неограниченной длины. В соответствии с граничным условием (2) на поверхности каждого модуля функция потока полоидального поля принимает постоянное значение одинаковое по абсолютному значению у всех модулей и меняющая свой 

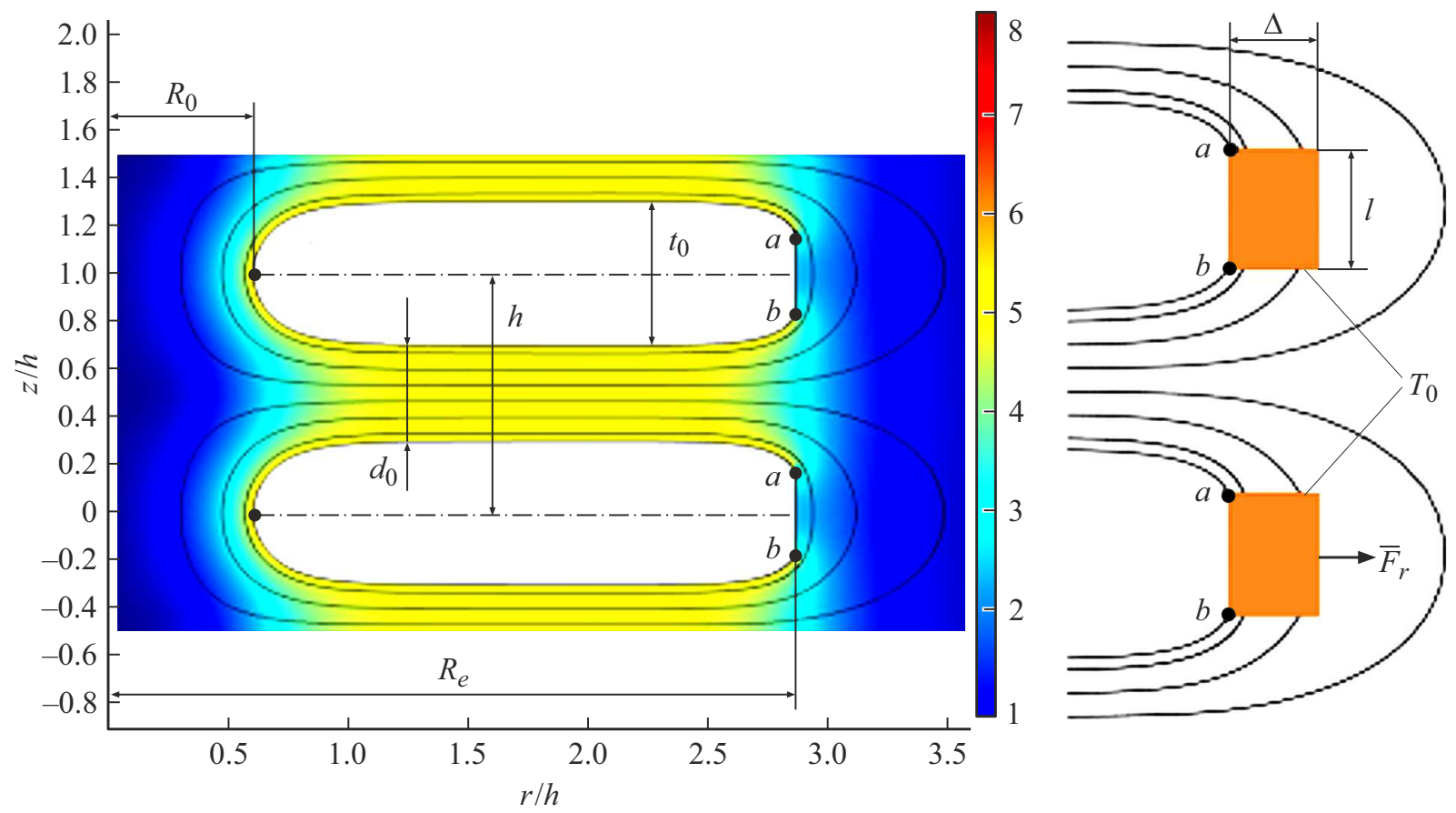

Рис. 3. Два элемента последовательности поперечных модулей неограниченной длины с частично уравновешенной тонкостенной обмоткой и бандажом $T_{0}$. На шкале цветов отложены значения безразмерной величины $B r h / \psi_{0}$.
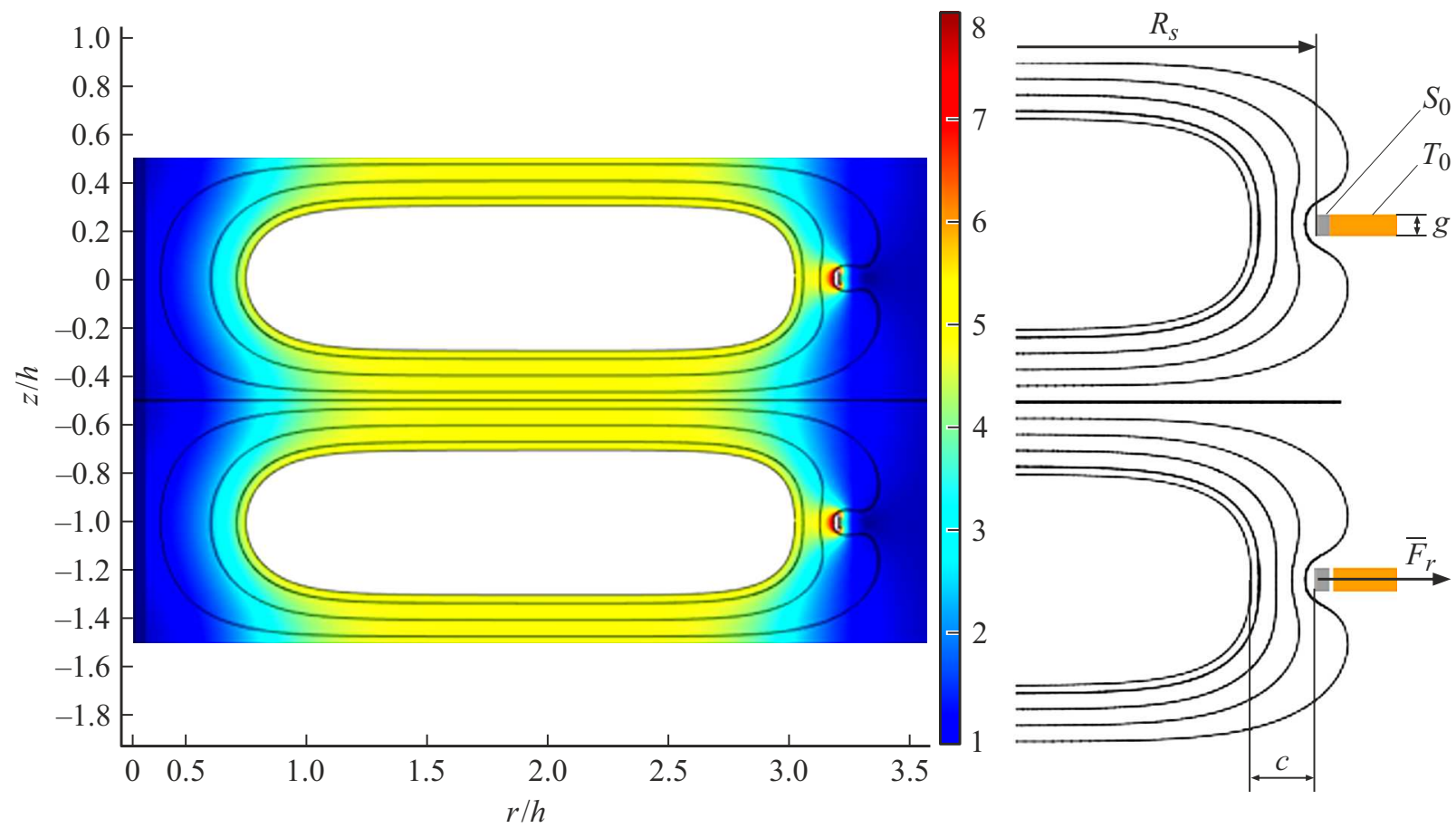

Рис. 4. Два элемента последовательности поперечных модулей неограниченной длины с полностью уравновешенной тонкостенной обмоткой, короткозамкнутым кольцом (экраном) $S_{0}$ и бандажом $T_{0}$.

знак при переходе от каждого модуля к следующему. При этом на плоскостях, являющихся границами раздела элементов системы, выполняется условие $\psi_{P}=0$. Далее будут рассмотрены конфигурации, у которых шаг системы плоских модулей в несколько раз меньше их внешнего радиуса. Поэтому вид фигур равновесия в окрестности внутреннего радиуса определяется выбором отношения $R_{0} / h$, где $h-$ шаг системы (рис. 3). У модулей двух типов, показанных на рис. 3,4 отличаются лишь конфигурации правых, удаленных от 
оси частей. На ближнем к оси краю каждого из этих модулей в точке $C\left(r=R_{0}\right)$ индукции азимутального и полоидального полей принимают абсолютные значения $B(C)=\mu_{0}|i| 2 \pi R_{0}=\mu_{0}\left|\psi_{i}\right| / R_{0}$, а в других точках уравновешенной части границы имеем в соответствии с условием (3)

$$
B_{p}=B_{\varphi}=G_{0} / r
$$

где $G_{0}=B(C) R_{0}$. При выбранном произвольно значении функции потока на границе модуля $\left|\psi_{P}\right|=\psi_{0}$ должна быть рассчитана конфигурация модуля, на части границы которого поддерживается постоянство произведения $r B=G_{0}$. При удалении от оси модуль приобретает форму плоского диска толщиной $t_{0}$, отделенного зазорами $d_{0}=\left(h-t_{0}\right)$ от соседних модулей. В предельном случае плоского поля, когда выполнено условие $R_{0} / h \gg 1$, это отношение принимает значение $t_{0} / h=1 / 2$. В средней части границы поперечного модуля, вдали от его краев, имеет место зависимость $B_{p}=2 \psi_{0} / r d_{0}$, таким образом:

$$
G_{0}=2 \psi_{0} / d_{0}
$$

На рис. 3 показаны модули первого типа, у которых условие локального равновесия выполнено на границе за исключением участка в виде кольца шириной $l=a b$ с радиусом $R_{e}$. На этом участке условия равновесия не выполняются, магнитное давление азимутального поля выше, чем полоидального, и для удержания обмотки необходим диэлектрический бандаж толщиной $\Delta$. При неизменных относительных размерах вся рассматриваемая магнитная система (включая набор поперечных модулей и концевые части) характеризуется двумя исходными параметрами: функцией потока $\psi_{0}$ и шагом $h$. Энергия магнитного поля одного модуля $W_{1}$ с некоторой погрешностью может быть рассчитана без учета краевых эффектов по формуле, основанной на допущении, что изменение индукции полоидального и азимутального полей в области $R_{0}<r<R_{e}$ соответствует зависимости (11):

$$
W_{1}=\int_{R_{0}}^{R_{e}} \frac{G_{0}^{2} \pi h r d r}{\mu_{0} r^{2}}=W_{0}\left(\frac{h}{d_{0}}\right)^{2} \ln \frac{R_{e}}{R_{0}},
$$

где $W_{0}=\left(4 \pi \psi_{0}^{2}\right) /\left(\mu_{0} h\right)=10^{7} \psi_{0}^{2} / h-$ характерная энергия магнитной системы.

Точное значение энергии удобно представить в виде $W_{1}=\lambda W_{0}\left(h / d_{0}\right)^{2} \ln \left(R_{e} / R_{0}\right)$. Близкий к единице поправочный множитель $\lambda$ может быть найден путем численного расчета поля. На неуравновешенном участке границы в виде кольца шириной $l$ и длиной $2 \pi R_{e}$ магнитное давление азимутального поля с индукцией $B\left(R_{e}\right)=G_{0} / R_{e}$ приводит к появлению радиальной силы $\mathbf{F}_{r}$. Пренебрегая на этом участке действием более слабого полоидального поля, можно рассчитать абсолютное значение силы, приходящейся на единицу длины кольца, считая, что индукция азимутального поля убывает согласно (11):

$$
F^{\prime}=F_{r} / 2 \pi R_{e}=Q l B^{2}(C) R_{0}^{2} / 2 \mu_{0} R_{e}^{2}=2 Q \frac{\psi_{0}^{2} l}{\mu_{0} d_{0}^{2} R_{e}^{2}} .
$$

В этой формуле $Q-$ безразмерный множитель, определяемый относительными размерами магнитной системы. Для удержания обмотки с неуравновешенным участком границы необходимо использовать бандаж $T_{0}$ (рис. 3) с площадью поперечного сечения $S$. При условии $R_{e} \gg S^{1 / 2}$ в бандаже имеет место напряжение $\sigma_{\varphi} \approx F^{\prime} R_{e} / S$. В рассматриваемом случае оно близко к эквивалентному напряжению $\sigma_{M}$, рассчитанному по формуле фон Мизеса. Далее можно рассчитать массу бандажа, выполненного из материала с заданным механическим напряжением $\sigma_{M}$

$$
M_{1}=2 \pi \gamma R_{e} S=\frac{2 \pi \gamma R_{e}^{2}}{\sigma_{M}}=M_{0} Q\left(\frac{h}{d_{0}}\right)^{2}\left(\frac{l}{h}\right),
$$

где $\gamma$ плотность материала бандажа, $M_{0}=\left(4 \pi \psi_{0}^{2} / \mu_{0} h\right)\left(\gamma / \sigma_{M}\right) \quad$ - характерная масса магнитной системы. При заданной длине участка $l$, воспринимающего магнитное давление, масса бандажа не зависит от радиуса $R_{e}$ и площади его сечения подобно тому, как было показано в аналогичном примере еще в книге Лонгмайра [2]. Используя формулы (12), (14), находим приближенное значение вириального коэффициента поперечного модуля

$$
\theta_{1}=\frac{W_{1} \gamma_{1}}{M_{1} \sigma_{M}}=\Lambda \ln \frac{R_{e}}{R_{0}}
$$

В системе первого типа (рис. 3) безразмерный параметр $\Lambda=\lambda h / l Q$ определяется относительными размерами системы, которые характеризуются параметром $R_{0} / h$. Принимая $\lambda=1$ и $Q=1$, находим оценочное значение числа $\Lambda=h / l$.

Конфигурация равновесной фигуры формируется в ходе численного расчета. Параметры $\psi_{0}$ и $h$ выпадают из формулы для отношения энергии к массе. Поэтому они могут выбираться произвольно при численном расчете. В рассматриваемых примерах они принимались равными единице. Следует отметить, что по мере увеличения отношения $R_{0} / h$ равновесная фигура становится все более близкой к рассчитанной для плоского поля. Толщина такой фигуры $t_{0}$ равна половине шага $h$, а длина $l$ неуравновешенного участка равна нулю $[16,17]$. Таким образом, при увеличении указанного отношения масса бандажа становится сколь угодно мала, а коэффициент $\theta_{1}$, рассчитанный для элемента системы неограниченной длины, может быть сколь угодно велик. Это утверждение теряет силу для систем с ограниченным числом элементов, у которых в указанном предельном случае масса определяется концевыми частями магнитной системы.

Построение фигур равновесия описанным в Приложении методом итераций позволяет с приемлемой точностью выполнить оба условия на внешней границе 
модуля. Подтверждением этому является пример модуля первого типа, приведенный на рис. 3. При выбранном значении $R_{0} / h=0.75$ отношение радиусов равновесной фигуры составляет $R_{e} / R_{0}=2.86$. На уравновешенном участке границы с погрешностью менее $3 \%$ выполняется условие $B_{p} r=4.2 \psi_{0} / h$. Возможно более точное построение, но оно мало сказывается на результатах расчета энергии магнитного поля и массы короткозамкнутого проводника, воспринимающего нагрузку. В результате расчета найдены толщина модуля $t_{0}$, половина зазора между модулями $d_{0}=h-t_{0}$, длина уравновешенного участка $l$, индукция в точке $C$. Значения соответствующих безразмерных параметров составляют: $d_{0} / h=0.42, t_{0} / h=0.58, l / h=0, B(C)=6.35 \psi_{0} / h^{2}$. При этом оценочное значение параметра $T=h / l=2.94$. Ему соответствует значение вириального параметра $\theta_{1} \sim 8.4$. Расчет поля и силы с помощью программы Comsol Multiphysics позволяет найти численные значения безразмерной энергии и массы одного модуля первого типа: $W_{1} \sim 5 W_{0}, M_{1} \sim 0.62 M_{0}$, что дает близкое значение вириального коэффициента: $\theta_{1} \sim 8.06$.

Второй тип фигур равновесия имеет место в случае, когда квазибессиловая обмотка с внешним радиусом $R_{e}$ находится в поле коаксиального короткозамкнутого контура (диамагнитного экрана). Таким контуром может быть, например, тонкое кольцо прямоугольного сечения $S_{0}$ с бандажом $T_{0}$ (рис. 4). В этом случае обмотка полностью уравновешена, нагрузка воспринимается материалом бандажа, удерживающего кольцо. Расчеты этой системы выполнены с использованием граничного условия $\psi_{P}=0$ на поверхности кольца. В импульсном поле это соответствует условию резко выраженного скин-эффекта. При большом отношении радиусов $R_{e} / R_{0}$ и выбранном значении $R_{0} / h=0.75$ конфигурация левой части фигуры равновесия и отношение $d_{0} / h$ остаются такими же, как в системе рис. 3. Для указанного модуля построены конфигурации равновесной границы в присутствии экранов различного радиуса $R_{S}$ с различными длинами $g$, рассчитана энергия магнитного поля $W_{2}$, полная радиальная сила $\mathbf{F}_{r}$, масса бандажа

$$
M_{2}=\gamma F_{r} R_{S}, \sigma_{M}
$$

и найдено значение вириального коэффициента одного элемента

$$
\theta_{2}=\frac{W_{2}}{R_{S} F_{r}}
$$

Равновесие модуля в примере, показанном на рис. 4 , имеет место, если $R_{S}=3.2 h, c=0.18 h, g=0.05 h$. Небольшие вариации указанных размеров мало влияют на численное значение энергии и массы. Они принимают значения $W_{2} \sim 4.3 W_{0}$ и $M_{2} \sim 0.18 M_{0}$. Масса бандажа во втором типе магнитной системы существенно меньше, чем в первом. Это объясняется тем, что даже при небольшом сечении короткозамкнутого экрана его наличие позволяет при соответствующем выборе радиуса $R_{S}$ построить границу с требуемыми граничными условиями. Вириальный коэффициент модуля второго типа в данном примере принимает существенно большее значение, чем в первом: $\theta_{2} \sim 23.8$.

\section{3. Построение концевых частей магнитной системы}

Рациональное построение концевой части имеет важное значение, поскольку она обеспечивает равновесие магнитной системы в целом. Вместе с тем в этой области может быть сосредоточена значительная часть энергии магнитного поля и массы магнитной системы. Конфигурация концевой части представлена на рис. 5. Она представляет собой комбинацию однотипных элементов. Каждый $n$-й элемент состоит из продольного и поперечного модулей с номерами $n^{\prime}$ и $n^{\prime \prime}$. На рис. 5 приведен пример системы с тремя такими элементами. Продольный и поперечный модули каждого элемента разделены узкими зазорами. Наличие этих зазоров практически не влияет на поле, силовые линии которого огибают границы модулей. Каждый продольный модуль характеризуется внутренним радиусом $R_{n}$ и внешним $R_{n}^{\prime}$. Границей концевой части целесообразно считать плоскость $Y$, делящую крайний из системы чередующихся поперечных модулей с номером 0 на две равные части. Половина крайнего из этих модулей может быть включена в состав концевого элемента. Функцию потока полоидального поля на указанной границе можно считать равной нулю.

Расстояния между модулями соседних элементов, форма краевых участков и конфигурация „скругленных“ границ на стыке продольных и поперечных модулей каждого элемента должны быть рассчитаны так, чтобы на границах были выполнены упомянутые выше условия постоянства функции потока $\psi_{i}$ и произведения $B_{p} r$.

Первым шагом при построении равновесной концевой части системы является расчет внутреннего радиуса первого продольного модуля $R_{1}$ и построение конфигурации области между крайним элементом последовательности поперечных модулей и поперечным модулем $1^{\prime \prime}$ (рис. 5). Далее будем исходить из допущения, что радиальный размер этих модулей много больше ширины зазора между ними $d_{1}$. Это позволяет считать одинаковыми значения индукции полоидального поля $B_{P}$ в точках $m_{0}$ и $n_{1}$ с одинаковыми радиальными координатами $r$ в средней части зазора $d_{1}$. Параметр $G=B_{p} r$ в обеих точках принимает одинаковое значение $G_{0}=2 \psi_{0} / d_{0}$. Разность функций потока полоидального поля в указанных точках можно представить в виде $\psi_{0}-\psi_{1}=B_{p}\left(M_{0}\right) r d_{1}=G_{0} d_{1}=2 \psi_{0} d_{1} / d_{0}$. В точке $a$ на границе продольного модуля $1^{\prime}$, находящейся в области, где полоидальное поле практически однородно, функция потока принимает значение $\psi_{1}=-(1 / 2) B_{1} R_{1}^{2}=-(1 / 2) G_{0} R_{1}=-\psi_{0} R_{1} / d_{0}$. Здесь учтено, что вектор индукции $\mathbf{B}_{1}$ в указанной области имеет другое направление, чем вектор $\mathbf{B}_{0}$, а параметр $G_{0}$ 


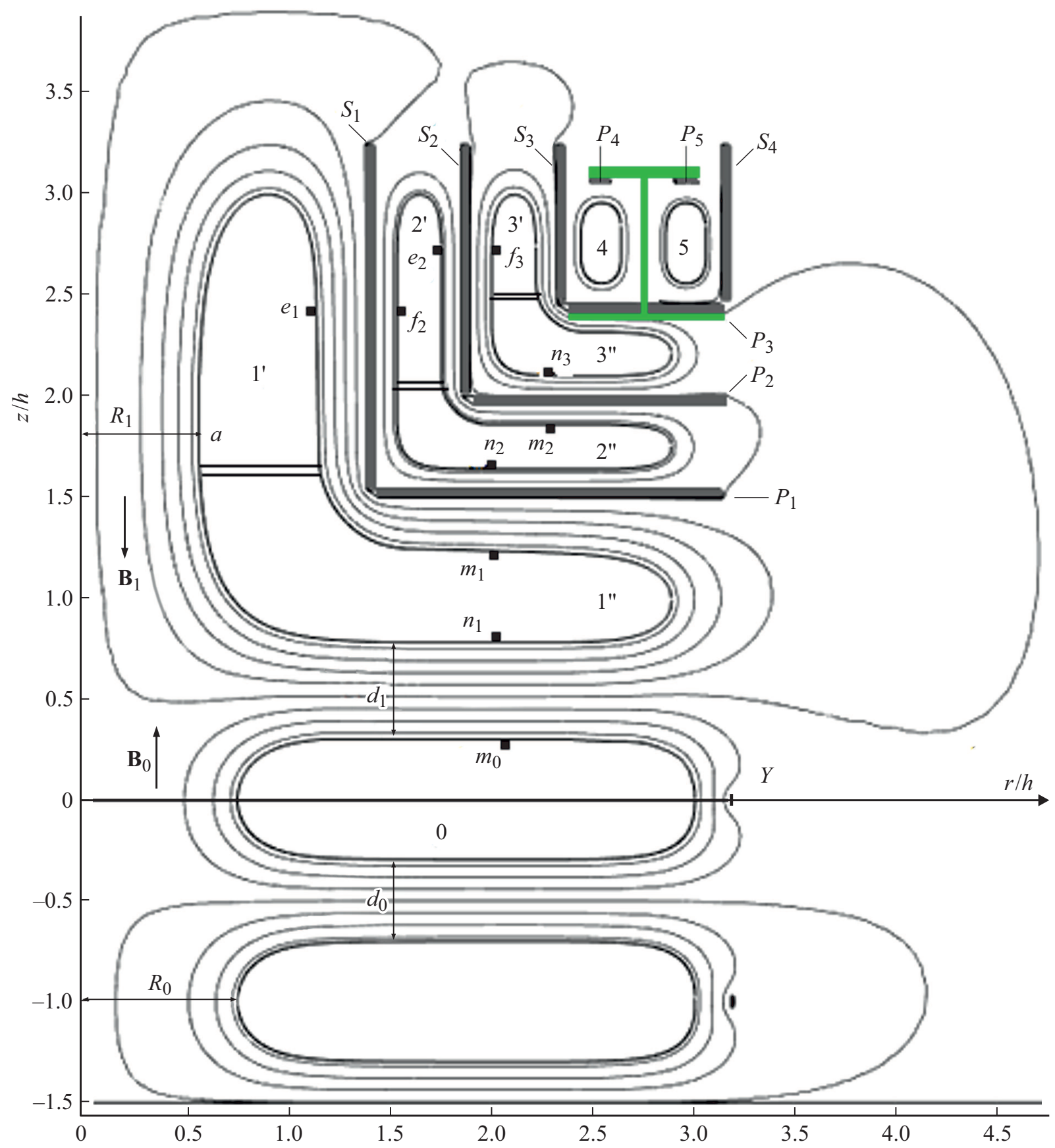

Рис. 5. Концевая часть магнитной системы конечной длины с комбинацией продольных и поперечных модулей и прилегающая к ней часть с последовательностью поперечных модулей.

сохраняет такое же значение, как и в соседнем элементе. Из приведенных уравнений следует выражения для внутреннего радиуса продольного модуля $1^{\prime}$ и функции потока $\psi_{1}: R_{1}=2 d_{1}-d_{0}, \psi_{1}=\psi_{0}\left(1-2 d_{1} / d_{0}\right)$. При построении границы в ходе итераций варьируется значение зазора $d_{1}$. При определенном значении $d_{1}$ и соответствующих значениях радиуса $R_{1}$ и функции потока $\psi_{1}$ формируется фигура, на границе которой наряду с условием $\psi=$ const $=\psi_{1}$ выполнено второе граничное условие $R_{1} B_{1}=G_{0}$. На рис. 5 представлен результат такого построения. При найденных значениях $d_{0} / h=0.42, d_{1} / h=0.49, R_{i, 1} / h=0.56$ функция потока на границе первого модуля и индукция в зазоре принимают значения $\psi_{1}=-1.33 \psi_{0}, B_{1}=-8.5 \psi_{0} / h^{2}$.

В концевой части помимо продольных и поперечных модулей размещены цилиндрические и плоские тонкостенные короткозамкнутые экраны $S_{n}$. При отсутствии экранов условие (4) не может быть выполнено на всей границе зазора между соседними модулями. Действительно, значения индукции в точках $m_{n}$ и $n_{n+1}$, одинаково 
удаленных от оси, и лежащих на границах соседних поперечных модулей с номерами $n$ и $n+1$, равны. Вместе с тем на этих частях границ равны и значения произведений $B_{p} r$. Однако при равенстве индукций в точках $e_{n}$ и $f_{n+1}$, находящихся на границах соседних продольных модулей с номерами $n$ и $n+1$, значения указанного произведения в этих точках различны, поскольку указанные точки находятся на различных расстояниях $R_{n}^{\prime}$ и $R_{n+1}$ от оси. Следовательно, необходимо создать систему с различающимися значениями индукции на границах соседних продольных модулей. Для этого между указанными модулями размещены короткозамкнутые тонкостенные цилиндры (экраны) $S_{n}$. Они показаны жирными линиями на рис. 5. Расчеты концевой части магнитной системы выполнены при допущении того, что на границах всех экранов функция потока полоидального поля постоянна и принимает значение $\psi_{p}=0$. Магнитный поток огибает каждый модуль. При этом функция потока полоидального поля и параметр $G_{n}$ определяются следующими выражениями:

$$
\psi_{n}=\frac{G_{n}}{2 R_{n}}\left[R_{n}^{2}-R_{S, n-1}^{2}\right]=\frac{G_{n}}{2 R_{n}^{\prime}}\left[R_{S, n}^{2}-R_{n}^{\prime 2}\right]
$$

где $R_{n}$ - внутренний, $R_{n}^{\prime}$ - внешний радиусы продольного модуля, $R_{S, n-1}, R_{S, n}-$ радиусы экранов, между которыми расположен данный модуль. Соотношения между радиусами соседних модулей и радиусами экранов должны соответствовать условиям существования конфигураций, у которых условия равновесия выполнены на всей границе каждого продольного модуля, включая ее торцевую часть. В рассматриваемом примере системы, состоящей из трех двухмодульных элементов, при выбранном значении радиуса первого продольного модуля $R_{1}=0.56 h$ внешний радиус этого модуля $R_{1}^{\prime}$ и радиус экрана $R_{S, 1}$ определяются теми же соотношениями, которые приведены выше для уравновешенной тонкостенной обмотки в экране: $R_{1}^{\prime}=2.12 R_{1}=1.19 h$, $R_{S, 1}=2.57 R_{1}=1.44 h$. Следующий продольный модуль расположен между двух экранов с радиусами $R_{S, 1}$ и $R_{S, 2}$. Продольные модули являются примерами фигур равновесия, расположенных между двумя коаксиальными экранами - внутренним и внешним (рис. 2,a). При заданном значении отношения внутреннего радиуса $n$-й обмотки к радиусу внутреннего экрана $R_{n} / R_{S, n-1}$ конфигурация модуля с постоянным значением функции потока и произведения $B_{p} r$ может быть построена лишь при вполне определенном значении отношений внутреннего, внешнего радиусов модуля и радиуса внешнего экрана $R_{S, n}$ к радиусу внутреннего экрана $R_{S, n-1}$. Значения этих отношений приведены на рис. $2, b$. В рассматриваемом примере в первом приближении толщины второго и третьего модулей выбраны одинаковыми, а зазоры между этими модулями и экранами выбраны равными половине ширины модуля. Такие соотношения размеров имеют место в плоском поле. Выбор их в данном случае обоснован тем, что толщины зазоров малы по сравнению с

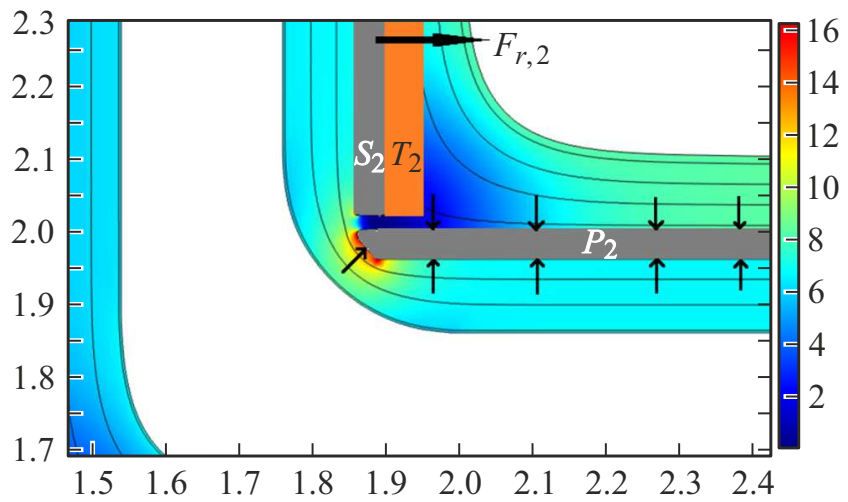

Рис. 6. Фрагмент концевой части магнитной системы, расположенной между вторым и третьим модулями. Стрелками обозначены направления сил, воздействующих на плоский проводник $P_{2} . \mathbf{F}_{r, 2}-$ сила, воздействующая на цилиндрический экран $S_{2}$ и передаваемая бандажами $T_{2}$.

внутренними радиусами указанных модулей. Этот выбор использован в качестве первого приближения. Далее проводится уточненное построение границ модулей с использованием метода итераций.

Аксиальные силы в концевой части системы должны быть уравновешены. Конфигурация продольных модулей удовлетворяет этому условию, а для поддержания равновесия экранов использованы дополнительные короткозамкнутые тонкостенные плоские проводники $P_{n}$, расположенные между поперечными модулями. Размещение этих проводников позволяет избежать появления аксиальной силы, воздействующей на цилиндрические экраны, но при этом формируется небольшая аксиальная сила, воздействующая на плоскую часть проводника $P_{n}$ и его краевую часть (рис. 6).

Указанная сила может быть уравновешена магнитным давлением поля в зазоре между поперечным модулем и указанным проводником. Этого можно добиться путем соответствующего выбора значения функции потока полоидального поля на границе модуля. Вместе с цилиндрическими экранами плоские проводники разбивают расчетную область на автономные фрагменты со слабой магнитной связью между ними. Знаки функции потока на границах соседних модулей и значения произведения $B_{p} r=G_{n}=$ const различны. В каждом фрагменте производится построение методом итераций „скругленных“ участков границ в местах стыковки продольных и поперечных модулей с сохранением на этих участках условия (4). В построенной описанным образом системе цилиндрические экраны $S_{k}$ не уравновешены и должны удерживаться бандажами $T_{k}$, толщины которых определяются допустимым значением механического напряжения, определяемого разностью магнитного давления по обе стороны экрана. При рациональном выборе конфигурации модулей длины цилиндрических экранов и, следовательно, массы бандажей могут быть достаточно малы. В идеализированной системе можно не 


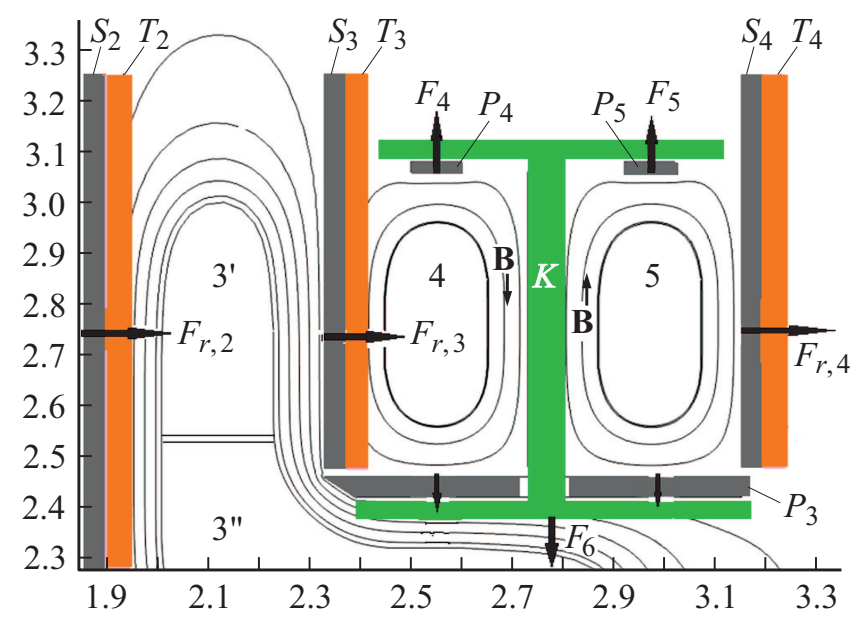

Pис. 7. Угловой элемент концевой части магнитной системы с экранами $S_{k}$, плоскими кольцами $P_{k}$ и бандажами $T_{k}$.

считаться с массой плоских проводников, разгруженных от интегральных осевых сил. При этом допущении лишь масса бандажей, удерживающих цилиндрические экраны, фигурирует при расчете вириального коэффициента концевых частей. В состав энергии и массы концевой части входит также энергия и масса половины крайнего из рассмотренной выше системы поперечных модулей, обозначенного символом 0 на рис. 5 .

Радиальные границы показанных на рис. 5 поперечных модулей $1^{\prime \prime}, 2^{\prime \prime}, 3^{\prime \prime}$ уравновешены путем выбора их конфигурации. Еще одним элементом концевой части является фрагмент, включающий в себя два коротких продольных модуля 4 и 5 , короткозамкнутые цилиндрические экраны $S_{3}$ и $S_{4}$, плоские короткозамкнутые проводники $P_{3}-P_{5}$ (рис. 7). Путем пробных расчетов выбирается такое расположение этих проводников, которое дает возможность построить методом итераций модули 4 и 5, на границе которых выполнено граничные условия (3) и (5). Цилиндрические экраны $S_{3}$ и $S_{4}$ и плоские короткозамкнутые проводники $P_{3}-P_{5}$ подвержены воздействию аксиальных электромагнитных сил. Эти усилия воспринимаются диэлектрическим телом, состоящим из двух плоских дисков и соединяющего их кольца $K$. На верхний диск передаются аксиальные силы $\mathbf{F}_{4}$ и $\mathbf{F}_{5}$, воздействующие на проводники $P_{4}$ и $P_{5}$. Равнодействующая этих сил приложена к верхней части кольца и направлена по оси $z$ в положительном направлении. К нижней части кольца приложена сила $\mathbf{F}_{6}$, воздействующая на проводник $P_{3}$ и нижний диск. В рассмотренном примере функция потока на границе третьего модуля $\psi_{3}=-0.84 \psi_{0}$ рассчитана так, что эта сила направлена в отрицательном направлении и численно равна сумме $F_{4}+F_{5}$. Таким образом, вся совокупность проводников, представленных на рис. 7, уравновешена в аксиальном направлении. В кольце $K$ с площадью поперечного сечения $S$ возникают растягивающее напряжение. При одноосном растяжении его абсолютное значение $\left|\boldsymbol{\sigma}_{z}\right|=\sigma_{M}=F_{6} / S$. Оно определяет
Таблица 1. Нормализованные значения сил, воздействующих на экраны, и произведения сил на радиусы экранов

\begin{tabular}{c|c|c|c|c|c}
\hline Номер экрана & 0 & 1 & 2 & 3 & 4 \\
\hline$\left(\mu_{0} h^{2} / 4 \pi \psi_{0}^{2}\right) F_{r, k}$ & 0.19 & 2.20 & 0.73 & 3.08 & 0.78 \\
\hline$\left(\mu_{0} h / 4 \pi \psi_{0}^{2}\right) R_{r} F_{r, k}$ & 3.20 & 1.39 & 1.92 & 7.07 & 2.51
\end{tabular}

площадь сечения кольца. Радиальные силы, воздействующие на плоские диски $P_{3}, P_{4}, P_{5}$, пренебрежимо малы. Пренебрегая массой этих тел, можно считать массу диэлектрического тела равной массе кольца $K$ :

$$
M_{K} \approx \gamma S l_{K}=\frac{\gamma F_{6} l_{K}}{\sigma_{M}}
$$

где $l_{K}$ - длина кольца $K$.

Дальнейший расчет выполнен для системы, в которой все короткозамкнутые проводники, испытывающие воздействие радиальных сил, удерживаются диэлектрическими бандажами. Плотность диэлектрического материала $\gamma$ принята одинаковой для всех цилиндрических бандажей системы и для кольца $K$. Бандажи показаны на части рисунков (рис. $1-4,6,7$ ). При расчете массы нагруженных элементов концевой части магнита $M_{3}$ можно пренебречь массой проводников, если их толщина мала по сравнению с толщиной диэлектрических бандажей, и использовать формулу (17):

$$
M_{3}=\frac{\gamma}{\sigma_{M}}\left(\sum R_{k} F_{r, k}+F_{6} l_{K}\right),
$$

где $R_{k}$ - радиусы всех тонкостенных цилиндрических экранов, $F_{r, k}$ - воздействующие на них радиальные силы.

Полоидальное и азимутальное поля концевой части магнитной системы рассчитаны с помощью программы Comsol Multiphysics, найдены энергия полоидального и азимутального магнитных полей концевой части $W_{3}$ и силы, фигурирующие в формуле (21). Как и при расчете системы $A$, приняты граничные условия $\psi_{0}=1$ и $h=1$. Результаты расчета безразмерных сил $\left(\mu_{0} h^{2} / 4 \pi \psi_{0}^{2}\right) F_{r, k}$ приведены в табл. 1.

Характерные параметры системы удержания угловых модулей 4 и 5 составляют $\left(\mu_{0} h^{2} / 4 \pi \psi_{0}^{2}\right) F_{6}=0.28$, $l_{K} / h=0.6$. По этим данным рассчитаны энергия, масса и вириальный параметр концевой части магнитной системы:

$$
\begin{gathered}
\left(\mu_{0} h / 4 \pi \psi_{0}^{2}\right) W_{3} \sim 38.2, \quad\left(\sigma_{M} \mu_{0} h / \gamma 4 \pi \psi_{0}^{2}\right) M_{3} \sim 16.2, \\
\theta_{3}=\frac{\gamma W_{3}}{\sigma_{M} M_{3}} \sim 2.35 .
\end{gathered}
$$

Вириальный коэффициент полной системы, включающей в себя $N$ чередующихся поперечных модулей 
Таблица 2. Вириальный коэффициент системы с различным числом поперечных модулей

\begin{tabular}{c|c|c|c|c|c}
\hline$N$ (число модулей $)$ & 1 & 10 & 20 & 30 & 40 \\
\hline$\theta_{\Sigma}$ (первый тип модулей) & 2.35 & 3.81 & 4.75 & 5.35 & 5.76 \\
\hline$\theta_{\Sigma}$ (второй тип модулей $)$ & 2.35 & 4.32 & 6.09 & 7.51 & 8.83
\end{tabular}

первого или второго типа, можно рассчитать по формуле

$$
\theta_{\Sigma}=\frac{\gamma\left[(N-1) W_{1,2}+2 W_{3}\right]}{\sigma_{M}\left[(N-1) M_{1,2}+2 M_{3}\right]}
$$

Число поперечных модулей в этой формуле уменьшено на единицу, поскольку энергия и масса одного из них учитываются при расчете концевых частей. Минимальное значение этого параметра, $\theta_{\Sigma}$ равное $\theta_{3}$, имеет место при $N=1$. В системе неограниченной длины $(N \gg 1)$ для двух рассмотренных выше видов поперечных модулей достигаются предельные значения вириальных коэффициентов $\theta_{\Sigma}=\theta_{1} \sim 8.06$ и $\theta_{2} \sim 23.8$.

Данные табл. 2 показывают, что при выбранных размерах элементов магнитной системы значения $\theta_{\Sigma}$, существенно превышающие единицу, достигаются при числе $N$ порядка нескольких десятков.

\section{Заключение}

Масса магнитной системы с проводниками, разгруженными от электромагнитных сил, определяется в основном не обмоткой, а теми элементами, которые подвержены воздействию этих сил. Такими элементами являются бандажи, удерживающие неуравновешенные части обмоток, и дополнительные короткозамкнутые экраны. Возможность снижения массы этих элементов рассмотрена в рамках идеальной системы с тонкой бессиловой обмоткой. Выполненные построения и расчеты показали эффективность использования системы с большим числом чередующихся модулей в сочетании с многомодульными концевыми частями специальной конфигурации. Применение комбинации продольных и поперечных модулей позволяет минимизировать массу элементов, нагруженных электромагнитными силами. Благодаря этому могут быть значительно увеличены значения вириального коэффициента, характеризующего отношение энергии к массе. Описанные методы построения фигур равновесия могут быть использованы при дальнейшей оптимизации аналогичных магнитных систем. Ключевым моментом при их построении является использование комбинации продольных и поперечных модулей. Вместе с тем предложенные конфигурации дают основу для построении магнитных систем с квазибессиловыми обмотками и расчета вириального коэффициента с учетом массы уравновешенных проводников.

\section{Приложение}

Построение свободной границы методом итераций

Итерационным путем производится решение задачи с двумя граничными условиями (2), (4), заданными на границе с неизвестной конфигурацией (задача со свободной границей $[20]$ ). Радиусы-вектора точек границы удобно представить в форме комплексных чисел: $p(s)=z(s)+i r(s)$, где $s-$ параметр - длина дуги, отсчитываемая от начала свободной границы с заданной координатой $p(0)=p_{0}$. Вектор магнитной индукции полоидального поля $\mathbf{B}(s)$ в этой точке также записывается в комплексной форме. Процесс итераций состоит из двух этапов.

Этап 1. На каждом шаге итераций выполняется расчет магнитостатической задачи с заданным граничным условием (2) путем решения интегрального уравнения первого рода На первом шаге форма границы задается начальным приближением, на каждом последующем используется граница, рассчитынная на предыдущем шаге. Вектор индукции $\mathbf{B}_{1}(s)=B_{1}(s) \exp \left(i \alpha_{1}\right)$ направлен по касательной к границе проводника, где $\alpha_{1}-$ угол направления касательной. Далее, в соответствии с условием равновесия, принимается $\left|B_{\varphi}(s)\right|=B_{1}(s)$ и путем усреднения функции $r\left|B_{\varphi}(s)\right|$ на границе длиной $l$ вычисляется постоянная $C_{2}$ :

$$
C_{2}=\frac{1}{l} \int_{l} r\left|B_{\varphi}(s)\right| d l(s) .
$$

Этап 2. Путем решения интегрального уравнений второго рода производится расчет магнитостатического поля с граничным условием (4), где постоянная $C_{2}$ определена на первом этапе. Вектор магнитной индукции на границе принимает новое значение $\mathbf{B}_{2}=B_{2} \exp \left(i \alpha_{2}\right)$ и изменяет свое направление на угол $\Delta \alpha=\alpha_{2}-\alpha_{1}$

Изменение угла означает, что нарушается граничное условие (2), согласно которому вектор магнитной индукции должен быть направлен по касательной к границе. Для выполнения условия (2) выбирается новое значение угла наклона касательной в каждой точке границы $\alpha^{\prime}=\alpha+\lambda \Delta \alpha$, где $\lambda-$ итерационный параметр, ускоряющий сходимость процесса: $1<\lambda<2$ При заданном значении угла наклона касательной к границе $d p / d s=\exp \left(i \alpha^{\prime}(s)\right)$ новая координата узла свободной границы с номером $k+1$ может быть рассчитана по формуле трапеций: $p_{k+1}=p_{k}+h_{k} \exp \left[0.5 i\left(\alpha_{k}^{\prime}+\alpha_{k+1}^{\prime}\right)\right]$, $k=0, \ldots, N-1$, где $N-$ число элементов свободной границы, $\alpha_{k}^{\prime}, \alpha_{k+1}^{\prime}-$ угол наклона касательной в начальной и конечной точке каждого элемента, $h_{k}$ - его длина. Выбор $h_{k}$ производится так, чтобы смещение узловых точек происходило в заданном направлении (обычно параллельно одной из координатных осей).

Итерационный процесс продолжается до достижения с заданной точностью условия (с заданной точностью): $\alpha_{2}-\alpha_{1}=0$. На примерах плоских задач, допускающих сравнение с аналитическим решением, подтверждена 
сходимость процесса итераций, исходящего из начального приближения, далекого от окончательного [20].

\section{Финансирование работы}

Работа выполнена при поддержке гранта Российского научного фонда № 18-19-00230.

\section{Конфликт интересов}

Авторы заявляют, что у них нет конфликта интересов.

\section{Список литературы}

[1] E.N. Parker. Phys. Rev., 109 (5), 1440 (1958).

[2] К. Лонгмайр. Физика плазмы (Атомиздат, М., 1966), $341 \mathrm{c}$.

[3] В.Д. Шафранов. В сб.: Вопросы теории плазмы, под ред. М.А. Леонтовича (Госатомиздат, М., 1963), с. 92.

[4] Б.А. Ларионов, Ф.М. Спевакова, А.М. Столов, Э.А. Азизов. В сб.: Физика и техника мощных импульсных систем, под ред. Е.П. Велихова (Энергоатомиздат, М., 1987), с. 66.

[5] Y.M. Eyssa, R.W. Boom. IEEE Trans. Magnet., MAG.17 (1), 460 (1981).

[6] W. Hassenzahl. IEEE Trans. Magnet., MAG.25(2), 1854 (1989).

[7] M.A. Hilal, O. Arici, M. Cuban. IEEE Trans. Magnet., MAG.21 (2), 1044 (1985).

[8] F.C. Moon. J. Appl. Phys., 53 (12), 9112 (1982).

[9] S. Nomura, T. Osaki, J. Kondoh, H. Tsutsui, S. Tsuji-Lio, Y. Sato, R. Shimada. IEEE Trans. Appl. Superconductivity, 9 (2), 354 (1999).

[10] S. Nomura, H. Tsutsui, N. Watanabe, C. Suzuki, S. Kajita, Y. Ohata, T. Takaku, E. Koizumi, S. Tsuji-Lio, R. Shimada. IEEE Trans. Appl. Superconductivity, 13 (2), 1852 (2003).

[11] S. Nomura, H. Tsutsui. 27 (4), 5700106 (2017).

[12] H. Kamada, A. Ninomiya, S. Nomura, T. Yagai, T. Nakamura. IEEE Trans. Appl. Superconductivity, 30 (4), 4600905 (2021).

[13] G.A. Shneerson, M.I. Dolotenko, S.I. Krivosheev. Strong and Superstrong Pulsed Magnetic Fields Generation (Berlin, De Gruyter, 2014), p. 429.

[14] G.A. Shneerson, A.P. Nenashev., A.A. Parfentiev., I.A. Vecherov., S.A. Shimanskiy. IEEE Transactions on Plasma Science. 46 (9), 3209 (2018).

[15] М.И. Гуревич. Теория струй идеальной жсидкости (Наука, М., 1979)

[16] А.Б. Новгородцев, Ф.Р. Фатхиев. Радиотехника и электроника. (5), 874 (1982).

[17] Г.А. Шнеерсон. Поля и переходные процессы в аппараmуре сверхсильных токов (Энергоатомиздат, М., 1992), 414 c. [G.A. Shneerson. Fields and Transients in Superhigh Pulse Current Devices (Nuova Science, NY., 1997), p. 561.]

[18] Э.Л. Амромин, Г.Н. Капорская, А.Б. Новгородцев, С.Л. Шишигин, Г.А. Шнеерсон. Электричество, (3), 40 (1989).

[19] С.Л. Шишигин. Электричество. (9), 51 (2008).

[20] Г.А. Шнеерсон, А.А. Парфентьев, В.В. Титков, С.И. Кривошеев, А.Д. Лагуткина, А.С. Немов, А.П. Ненашев, С.А. Шиманский. Письма в ЖТФ, (11), 40 (2021). 